\section{Hegab TMJ Splint (HTS)}

\section{Editorial}

Internal derangement of the temporomandibular joint (TMJ) is the main source of jaw pain, clicking, and/or crepitation and limitation of the mouth opening which resulted from the abnormal positional relationship of the disk relative to the condylar head, fossa, and/or articular tubercle. ${ }^{1,2}$ The term derangement refers to an alternation of the TMJ movement which involves to large degree the function of the articular disk. Consequently, the alteration refers to disk derangement. Despite its effectiveness, the occlusal splint considered as most common treatment modality for internal derangement of the temporomandibular joint. ${ }^{3}$

A wide variety of occlusal splint designs are known to be useful in the treatment of TMJ internal derangement. However; the results of the treatment of internal derangement of the temporomandibular joint using occlusal splint showed wide varieties among the studies. The reason behind that the selection of appropriate vertical thickness of occlusal splints based on personal experience and the use of the same vertical thickness in cases of disk displacement with and without reduction. ${ }^{4}$

Successful treatment of the TMJ internal derangement is based on understanding the biomechanical performance of the TMJ and subsequently to understand the biomechanical changes within the TMJ resulted from the occlusal splint to achieve harmonious relationships among teeth, joints, and muscles. As the disc displaced anteriorly, the elevator muscles continue to seat the condyle in the fossa resulting in decreasing the joint space and posterior-superiorly movement of the condyle. The aim of the splint is to move the condyle downward and foreword besides movement of the disk toward the normal position. These movements resulting in increasing the joint space, decreasing the joint pressure especially on the posterior attachment of the articular disk. Moreover; the vertical thickness of the occlusal splint return the normal physiologic function of the masticatory mechanism by relax

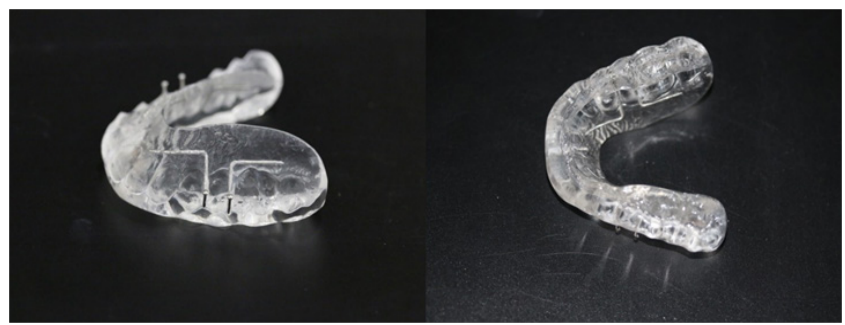

Figure I Hegab TMJ Splint (HTS).

\section{Acknowledgments}

None.

\section{Conflicts of interest}

The author declares that there is no conflict of interest.
Volume 10 Issue 2 - 2019

\author{
Ayman F Hegab \\ Department of Oral \& Maxillofacial Surgery, Faculty of Dental \\ Medicine,Al-Azhar University, Egypt
}

\begin{abstract}
Correspondence: Ayman F Hegab, Clinical Associate Professor, Department of Oral \& Maxillofacial Surgery, Faculty of Dental Medicine,Al-Azhar University, Cairo, Egypt, Email hegab@mail.com,Prof.aymanhegab@yahoo.com, dr.hegabacademy@aol.com,prof.hegab@azhar.edu.eg
\end{abstract}

Received: April 07, 2019 | Published: April 17, 2019

the masticatory muscles by decreasing the electrical muscle activity and re-establish the disk condyle relationship (disc re-capture). ${ }^{4}$

Hegab TMJ Splint (HTS) is Maxillary full-arch hard stabilization splints fabricated with fluid resin. The splint attached to the upper jaw using ball clasps without any extensions of the acrylic resin on the buccal or labial surface of the upper jaw. The retention of the splint to the upper jaw is coming only from the ball clasps. The occlusal splint has indentations on its occlusal surface to guide and hold the mandible in centric relation (Figure 1). The occlusal splint vertical thickness of $4 \mathrm{~mm}$ used for treatment of the cases with disk displacement with reduction (DDR-HTS4). While for cases of disk displacement without reduction, $6 \mathrm{~mm}$ vertical thickness of the occlusal splint was selected (DDNR-HTS6). The vertical thickness of the occlusal splint was measured at the molar area and the indentations help to achieve the Simultaneous equal contacts on all teeth (Figure 2). The selection of the vertical thickness of the occlusal splint based on evidence based study using the MRI to evaluate the movement changes within the TMJ with different splint thickness. ${ }^{4}$

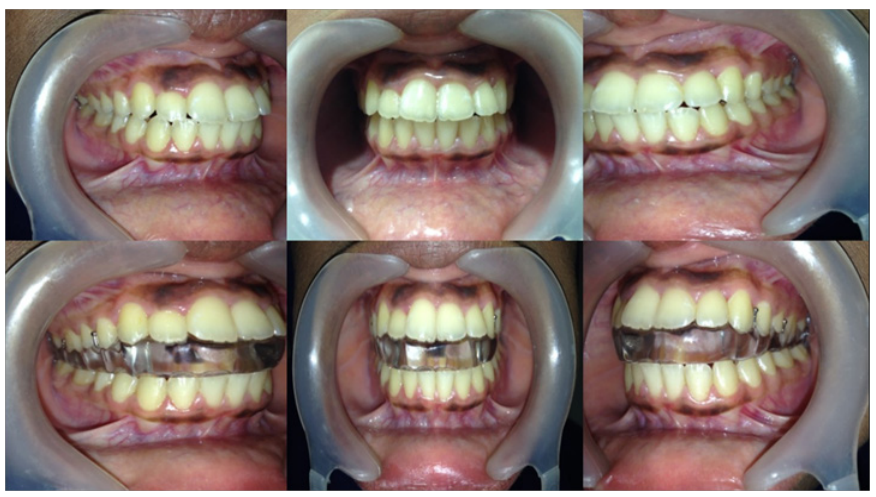

Figure 2 intraoral lateral and frontal view of the Hegab TMJ Splint (HTS).

\section{References}

1. Sanchez-Woodworth RE, Tallents RH, Katzberg RW, et al. Bilateral internal derangements of temporomandibular joint: evaluation by magnetic resonance imaging. Oral Surg Oral Med Oral Pathol. 1988;65(3):281-285.

2. Murakami S, Takahashi A, Nishiyama H, et al. Magnetic resonance evaluation of the temporomandibular joint disk position and configuration. Dentomaxillofac Radiol. 1993;22(4):205-207. 
3. Kostrzewa-Janicka J, Mierzwinska-Nastalska E, RolskiD, et al. Occlusal stabilization splint therapy in orofacial pain and tension type headache. Adv Exp Med Biol. 2013;788:181-188.
4. Ayman F Hegab, Ahmed Hossni Youssef, Hossam I. MRI based determination of occlusal splint thickness for temporomandibular joint disk derangement: a randomized controlled clinical trial. OOOO journal. 2018;125(1):74-87. 